John Damilakis

Judith E. Adams

Giuseppe Guglielmi

Thomas M. Link

\section{Radiation exposure in $\mathrm{X}$-ray-based imaging techniques used in osteoporosis}

Received: 10 December 2009

Revised: 8 April 2010

Accepted: 15 April 2010

Published online: 18 June 2010

(C) The Author(s) 2010.

This article is published with open access at

Springerlink.com

J. Damilakis $(-)$

Department of Medical Physics,

Faculty of Medicine,

University of Crete, P.O. Box 2208, 71003

Iraklion, Crete, Greece

e-mail: damilaki@med.uoc.gr

Tel.: +30-2810-392569

Fax: +30-2810-542095

\section{J. E. Adams}

Imaging Science and Biomedical

Engineering,

University of Manchester, Manchester, UK

\section{J. E. Adams}

Radiology Department,

Manchester Royal Infirmary, Manchester, UK

G. Guglielmi

Department of Radiology,

Scientific Institute Hospital San Giovanni

Rotondo, San Giovanni Rotondo, Italy
G. Guglielmi

University of Foggia, Foggia, Italy

T. M. Link

Department of Radiology

and Biomedical Imaging,

University of California, San Francisco, CA, USA

\begin{abstract}
Recent advances in medical $\mathrm{X}$-ray imaging have enabled the development of new techniques capable of assessing not only bone quantity but also structure. This article provides (a) a brief review of the current X-ray methods used for quantitative assessment of the skeleton, (b) data on the levels of radiation exposure associated with these methods and (c) information about radiation safety issues. Radiation doses associated with dual-energy $\mathrm{X}$-ray absorptiometry are very low. However, as with any X-ray imaging
\end{abstract}

technique, each particular examination must always be clinically justified. When an examination is justified, the emphasis must be on dose optimisation of imaging protocols. Dose optimisation is more important for paediatric examinations because children are more vulnerable to radiation than adults. Methods based on multi-detector CT (MDCT) are associated with higher radiation doses. New 3D volumetric hip and spine quantitative computed tomography (QCT) techniques and high-resolution MDCT for evaluation of bone structure deliver doses to patients from 1 to $3 \mathrm{mSv}$. Low-dose protocols are needed to reduce radiation exposure from these methods and minimise associated health risks.

Keywords Osteoporosis · Bone densitometry · DXA · QCT . Bone structure

\section{Introduction}

Osteoporosis is a systemic disorder of the skeleton that is characterised by a reduction in bone mass and deterioration of bone micro-architecture. Although the condition affects a higher percentage of women, it is now known that substantial bone loss occurs with advancing age in men. The importance of osteoporosis lies in the fact that osteoporotic bones are more fragile and susceptible to fracture than normal bones. Osteoporotic fractures usually occur in skeletal sites that are rich in trabecular bone. The most common low-energy fractures are those of the vertebrae, wrist and hip. Studies show that the number of osteoporotic fractures is increasing worldwide mainly due to the extension of the average lifespan. The total number of hip fractures in the European Union is estimated to increase from 414,000 in 2000 to 972,000 in 2050 [1].

A wide variety of methods for the non-invasive assessment of skeletal status have been developed, most of which are based on the use of ionising radiation. Although the ionising radiation doses incurred during $\mathrm{X}$-ray-based imaging techniques used in osteoporosis are relatively low, the use of radiological methods for the assessment of bone status has increased rapidly, and therefore merits attention with regard to radiation protection. The objective of this article was to briefly 
review the current X-ray methods used for the assessment of the skeleton, provide data that document the magnitude of radiation exposure and discuss radiation safety issues.

\section{Estimation of bone status using X-rays: current techniques}

Assessment of low-energy fractures

Spinal radiography is the most widely used imaging method for identification of vertebral fractures. Vertebral fractures on radiographs are not always reported and remain under-diagnosed radiologically with false negative rates up to $45 \%$ [2]. The assessment of vertebral fractures is possible using visual, morphometric and semiquantitative methods [3-6]. The method for identification of vertebral fractures using computational techniques has also been applied to spine images acquired by dual-energy X-ray absorptiometry (DXA). Vertebral fracture assessment (VFA) developed by DXA manufacturers provides information on the vertebral body heights and their ratios and the patient's fracture status is given. A recent study evaluated the utility of VFA to detect vertebral fractures [7]. Although the sensitivity of VFA was found to be less than that of radiography, in certain circumstances results support the use of VFA for the detection of prevalent vertebral fracture.

\section{Dual-energy X-ray absorptiometry (DXA)}

DXA technology has evolved from pencil beam to fan beam, allowing short acquisition time and improved image quality. In clinical practice, 'areal' bone mineral density $\left(\mathrm{BMD}_{\mathrm{a}} ; \mathrm{g} / \mathrm{cm}^{2}\right)$ assessment of lumbar spine (L1L4), proximal femur (femoral neck and total hip) and forearm (distal) is made by central DXA. Interpretation of $\mathrm{BMD}_{\mathrm{a}}$ measurements is based on the World Health Organisation (WHO) recommendations. Osteoporosis can be diagnosed if the value of $\mathrm{BMD}_{\mathrm{a}}$ is 2.5 or more standard deviations (SD) below the mean value of a young reference population ( $\mathrm{T}$ score at or below -2.5 ). Central DXA can also provide whole-body imaging for total and regional $\mathrm{BMD}_{\mathrm{a}}$, body composition (lean muscle and fat mass) and VFA. Total body imaging is useful for the assessment of the growing skeleton and forearm imaging can be performed in patients with hyperparathyroidism [8]. DXA at peripheral sites can be performed using either general purpose body DXA or smaller dedicated peripheral DXA for measurements in peripheral skeletal sites. Using device-specific thresholds peripheral DXA may play a role in identifying those at risk of osteoporotic fracture, especially when there is limited or no access to central DXA.
Quantitative computed tomography (QCT) using body CT

Using QCT, bone mineral density (BMD; $\mathrm{mg} / \mathrm{cm}^{3}$ ) measurements can be obtained in central and peripheral skeletal sites. Examinations are performed using an application-specific software package and a dedicated bone-equivalent calibration phantom imaged simultaneously with the patient to convert the CT numbers into bone-equivalent values $\left(\mathrm{mg} / \mathrm{cm}^{3} ; \mathrm{g} / \mathrm{l}\right)$. QCT requires a lateral scout image of the lumbar spine. A typical singleslice 2D QCT protocol consists of a 10-mm section in the mid plane of each of three or four adjacent vertebrae (T12, L1, L2 and L3) acquired with $80-\mathrm{kVp}$ tube potential and 125-mAs tube load. As this 2D technique has a limited precision, 3D volumetric QCT protocols have been developed based on multi-detector CT (MDCT) imaging. Using MDCT, 3D volume sets are acquired and from these BMD values and bone geometry can be measured $[9,10]$. In spine multi-detector QCT (MDQCT) two or three vertebrae are usually imaged, L1-L2 or L1-L3, to reduce dose. Hip MDQCT is capable of analysing the main regions of the hip i.e. the femoral neck, the trochanter and the intertrochanteric region.

\section{High-resolution CT imaging}

MDCT is not capable of depicting individual trabeculae. However, important information can be obtained from structure analysis of high-resolution image data. A recent study compared MDCT-derived apparent structure measures with high-resolution (HR) peripheral QCT (pQCT)-derived structure measures as the 'gold standard' using intact human cadaver forearm specimens [11]. Most MDCT-derived microarchitectural parameters correlated highly significantly with HR pQCT measures. This study shows that MDCT is capable of quantifying characteristics of the trabecular bone network in the radius [11]. Image processing techniques such as fuzzy distance transformation have been used to provide information on trabecular distance measurements in vertebrae imaged by HR CT [12]. Findings confirmed that this technique can potentially be used as a tool for monitoring osteoporosis treatment. However, currently, these techniques are limited to research applications.

\section{Peripheral QCT}

pQCT permits in vivo assessment of bone morphology and BMD at appendicular bones such as the distal radius and tibia. pQCT can be used simply for BMD and bone geometry, or in HR to provide information on trabecular bone structure. A recently developed device (Scanco, Bruttisellen, Switzerland) has an isotropic voxel size in the order of $80 \mu \mathrm{m}$, which allows direct or indirect evaluation of cortical and trabecular bone architecture. Specifically, assessment of parameters such as trabecular number, cortical thickness, trabecular thickness and 
porosity, and trabecular separation is possible with this technique. Recent studies have focused on HR pQCT imaging of bone microstructure in both adults and adolescents $[13,14]$.

\section{Use of DXA}

Use of DXA has increased steadily during recent years. Between 1996 and 2002, the number of DXA performed on all Medicare patients in the USA increased from 501,105 in 1996 to $2,195,548$ in 2002. This change represents a fourfold growth over 6 years, attributable to the extension of the average lifespan, increased public awareness of osteoporosis and advances in therapies [15]. The greatest increase in the DXA usage has been observed in the use of central densitometry [16]. These trends can be expected to continue for the next few years. In some countries DXA is only advocated in those with specific risk factors as population screening is not cost effective. In other countries there are national organisations that recommend primary adult screening with DXA for individuals at risk of osteoporosis. Thus, DXA is recommended for all women aged 65 years or over and for younger women with risk factors by the US National Osteoporosis Foundation and the US Preventive Services Task Force $[17,18]$. With the increasing use of bone densitometry, the necessity for justification and dose optimisation of examinations becomes a topic that deserves attention. As with other X-ray-based imaging methods, radiation dose from bone densitometry techniques that use ionising radiation (DXA, QCT) must be kept as low as reasonably achievable.

\section{Patient radiation doses}

Radiation dose quantities and units

Various radiation dose parameters are used in diagnostic radiology, the most commonly being absorbed dose and effective dose. Absorbed dose, expressed in grays (Gy), is a measure of the energy per unit of mass deposited in the tissue and organs of the body. Radiation dose from ionising radiation is frequently quantified in terms of the effective dose. The effective dose, expressed in sieverts (Sv), is calculated from information about absorbed doses to the organ or tissue exposed to X-rays and the relative radiation risk assigned to each of these organs or tissues. Appropriate weighting factors related to radiogenic risk for body organs and tissues have been published by the International Commission on Radiological Protection (ICRP) [19]. The effective dose was introduced to allow estimation of radiogenic risks when various organs receive different levels of dose. This commonly occurs with partial body exposures, which is always the case with
DXA. The effective dose is a useful quantity for comparison among different sources of ionising radiation, such as that from DXA and QCT or DXA and natural background radiation. The worldwide average effective dose from natural background radiation is $2.4 \mathrm{mSv} /$ year.

Two dosimetric quantities are utilised in CT, CT dose index (CTDI) and dose-length product (DLP) [20]. The CTDI represents the average absorbed dose, along the $\mathrm{z}$ axis, from a series of contiguous exposures. CTDI measurements are performed at the periphery $\left(\mathrm{CTDI}_{\mathrm{P}}\right)$ and at the centre $\left(\mathrm{CTDI}_{\mathrm{C}}\right)$ of cylindrical poly(methyl methacrylate) phantoms representing the human head and body by using a pencil ionisation chamber with a length of $100 \mathrm{~mm}$. From these measurements, a weighted CTDI $\left(\mathrm{CTDI}_{\mathrm{W}}\right)$ representing the average dose to a single slice can be derived as follows:

$\mathrm{CTDI}_{\mathrm{W}}=\frac{2}{3} \mathrm{CTDI}_{\mathrm{P}}+\frac{1}{3} \mathrm{CTDI}_{\mathrm{C}}$

To take into account the effect of pitch on radiation dose, CTDI volume $\left(\mathrm{CTDI}_{\mathrm{V}}\right)$ has been introduced for imaging performed in the spiral mode, which is defined as CTDI divided by pitch. $\mathrm{CTDI}_{\mathrm{V}}$ is the dose quantity displayed by the operator's console of most CT systems. The DLP is defined as the $\mathrm{CTDI}_{\mathrm{V}}$ multiplied by the imaging length. The SI unit for DLP is mGy $\mathrm{cm}$. Thus, DLP is an indicator of the integrated dose of an entire CT examination. Broad estimates of effective dose $E$ can be derived from DLP values using conversion coefficients: $E=\operatorname{DLP} \times k$ where $k$ is the normalised effective dose (mSv $\mathrm{mGy}^{-1} \mathrm{~cm}^{-1}$ ) that is a function of body region [21].

Radiation doses from techniques used to assess low-energy fractures

VFA is a low-dose technique with doses reported to be from 0.002 to about $0.05 \mathrm{mSv}$ [22-24]. Although spine radiography is the reference standard for the detection of vertebral fractures, VFA is associated with considerably lower exposure to the patient [25]. The dose from a lateral radiograph of the thoracic and lumbar spine is about $0.6 \mathrm{mSv}$ [22]. Obviously, the advantage of VFA with respect to radiation dose is doubtful when the examination is used in combination with spinal radiographs. MDCT is not performed specifically for vertebral fracture diagnosis. However, fracture assessment of the spine is possible without any additional radiation burden by routinely performing sagittal reformations in $3 \mathrm{D} \mathrm{CT}$ of the thorax and abdomen which have been performed for other clinical indications. In addition the lateral digital radiographs (scout views) may be used for fracture assessment. Radiographs are preferable to CT for routine diagnosis of vertebral fractures, partly because of the lower radiation dose. Bauer et al. [26] state that a low-dose CT protocol for visualisation of the spine is associated with a dose of $2.2 \mathrm{mSv}$ for men and $3.3 \mathrm{mSv}$ for women. 


\section{Radiation doses from DXA}

Several studies have reported doses to children and adults from DXA [27-34]. For the first-generation pencil-beam devices the effective dose was negligible i.e. about $0.001 \mathrm{mSv}$ for a spine and femur DXA. However, the doses are considerably higher for the fan-beam devices, especially for children and adolescents. Figure 1 shows effective doses to adult and paediatric patients from a spine and a hip DXA reported in a recent paper [33]. Dose figures were estimated by using the default adult imaging length of $20 \mathrm{~cm}$ for the spine and $15 \mathrm{~cm}$ for the hip. In general, the effective dose from a spine and hip DXA examination performed on a 5-year-old child is two to three times higher than the adult dose. This may be attributed to the fact that the exposure parameters and the image size are optimised for standard-sized adults. Paediatric doses can be reduced by adjusting image lengths to the size of the child's body. Table 1 shows typical organ and effective doses from fan-beam DXA and spine radiographs. DXA values are for Hologic DXA devices (Hologic Inc, Bedford, MA) examined by Blake et al. [33]. The patient dose will vary between DXA systems of different models and manufacturers depending on a number of variables, including differences in acquisition techniques and X-ray tube filtration. Doses for spine radiography were calculated by using the Monte Carlo N-
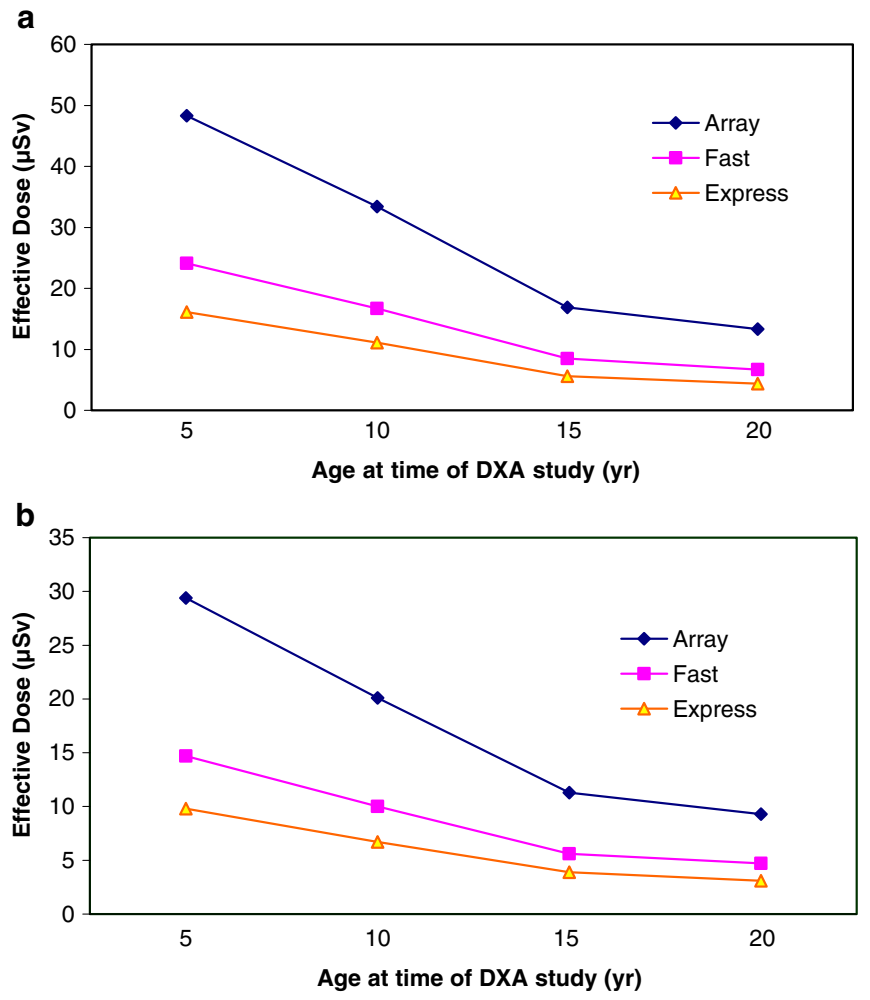

Fig. 1 Effective doses from a single DXA of the spine (a) or hip (b) as a function of patient age (adapted from Blake et al. [33]). Patient dose was estimated for Hologic DXA using three imaging modes: Array mode (60-s data acquisition time); Fast mode (30-s data acquisition time); and Express mode (10-s data acquisition time) particle code (MCNP, Los Alamos National Laboratory, Los Alamos, NM) and a mathematical phantom of human anatomy constructed with the BodyBuilder software package (BodyBuilder, White Rock Science, NM). Table 2 shows doses associated with various diagnostic X-ray examinations derived from the literature $[35,36]$. Patient effective doses from peripheral DXA are lower than $0.01 \mathrm{mSv}$ [37]. Whole-body DXA is an established procedure for the assessment of skeletal mineral status of the whole body and the measurement of body composition [38]. Effective doses for whole-body DXA examinations were found to be $0.0052,0.0048,0.0042$ and $0.0042 \mathrm{mSv}$ for a 5-, 10-, 15-year-old child and adult respectively for an examination performed on the Hologic Discovery A device. Corresponding values for the Hologic Discovery $\mathrm{W}$ were $0.0105,0.0096,0.0084$ and $0.0084 \mathrm{mSv}$ [33].

The patient radiation dose from a DXA examination depends on a number of parameters. The most important are the number of images, the size of the patient, the specific design of the device, beam filtration, the tube current $(\mathrm{mA})$, the tube potential $(\mathrm{kVp})$, the imaging speed and the imaging length and width. Most of these parameters cannot be controlled by the operator performing the DXA examination. However, it is important for the user to know that patient dose varies depending on the imaging mode for a specific examination (Fig. 1). Special attention to imaging protocols and radiation dose is needed when imaging children and adolescents. The use of a standardised and fixed clinical protocol designed for adults leads to unnecessary overexposure of children.

Although rare, DXA is occasionally performed on pregnant patients for the diagnosis or the differential diagnosis of pregnancy-associated osteoporosis. Pregnant patients receiving heparin may require $\mathrm{BMD}_{\mathrm{a}}$ estimation. The maximum conceptus dose during the first trimester associated with DXA performed using a pencil-beam device was found to be $0.0034 \mathrm{mGy}$ related to the scan of the hip [39]. In this study, conceptus doses from DXA performed during late pregnancy were found to be up to $0.0049 \mathrm{mGy}$. The highest dose was recorded for spinal imaging carried out during the third trimester of gestation. According to the ICRP, a dose to the conceptus below 100 mGy should not be considered a reason for terminating a pregnancy [40]. Because radiation dose to the unborn child from DXA is always less than $100 \mathrm{mGy}$, termination of pregnancy based on radiation risk is not justified. Although radiation dose to the conceptus is very low, DXA examinations on pregnant patients should be performed only when the expected benefits clearly exceed the reasonably suspected risks. When DXA is considered justified, the patient should be counselled before imaging on the actual dose received by the conceptus and the radiation risks involved.

\section{Radiation doses from QCT}

The patient dose from whole-body CT examination depends on a number of parameters including the technical features of the $\mathrm{CT}$, the selected acquisition 
Table 1 Effective and organ doses for DXA and spine radiographs

\begin{tabular}{llll}
\hline Examination & Effective dose $(\mathrm{mSv})$ & Organ dose (mGy) & Relevant organs \\
\hline Adult spine DXA & 0.013 & 0.003 & BM, ovaries \\
Adult hip DXA & 0.009 & 0.005 & LLI \\
$\begin{array}{l}\text { Paediatric spine DXA } \\
\text { (5-year-old child, scan length } 11.7 \mathrm{~cm})\end{array}$ & 0.027 & 0.008 & Ovaries \\
$\begin{array}{l}\text { Paediatric hip DXA } \\
\text { (5-year-old child, scan length } 9.0 \mathrm{~cm})\end{array}$ & 0.022 & 0.007 & Stomach \\
Paediatric spine DXA & & 0.015 & Testes \\
(10-year-old child, scan length $14.5 \mathrm{~cm})$ & 0.021 & 0.009 & LLI \\
Paediatric hip DXA & 0.018 & 0.006 & Ovaries \\
(10-year-old child, scan length $12.4 \mathrm{~cm})$ & & 0.005 & Stomach \\
Thoracic spine AP radiograph & 0.4 & 0.010 & Testes \\
Thoracic spine LAT radiograph & 0.3 & 0.008 & LLI \\
Lumbar spine AP radiograph & 0.7 & 1.2 & Lungs \\
Lumbar spine LAT radiograph & 0.3 & 2.5 & Lungs \\
\hline
\end{tabular}

Paediatric doses are given for scans lengths adjusted to the size of the child's body

$A P$ anterior-posterior, $L A T$ lateral, $B M$ bone marrow, $L L I$ lower large intestine

parameters and the size of the patient [41]. Thus, patient doses from CT differ significantly among study sites and CT systems. Dose depends strongly on the selected X-ray spectrum (i.e. the tube potential and the filtration), the tube current $(\mathrm{mA})$ and examination time. Compared with $\mathrm{CT}$ as an imaging technique, spinal single-slice 2D QCT examinations use low-exposure parameters, i.e. $80-\mathrm{kV}$ tube voltage and $120-140 \mathrm{mAs}$. This may be attributed to the fact that the high contrast between bone and surrounding structures allows BMD evaluation even with high noise levels. Table 3 shows radiation doses of optimised QCT protocols derived from the literature $[9,30,42-44]$.

Radiation doses from high-resolution CT imaging (HRCT)

With helical MDCT, a volume of tissue is imaged to capture a texture of the trabecular bone at skeletal sites such as the spine and the hip. The advantage is that a full data set is obtained, providing important information about bone structure. However, HR CT is associated with a considerably higher radiation dose compared with the Xray examinations commonly used in routine clinical practice for the estimation of bone status. Indeed, compared with the 0.06 to $0.3 \mathrm{mSv}$ patient effective dose associated with QCT applied to 2D slices in the lumbar spine, studies show that protocols used to examine vertebral microstructure using HR MDCT provide an effective dose of about $3 \mathrm{mSv}[45,46]$. This dose is similar to that delivered to the patient from 3D QCT of the hip (Table 3).
Radiation doses from $\mathrm{pQCT}$

Peripheral QCT is associated with a low radiation dose because radiosensitive organs are distant from the primarily exposed area. Studies show that the effective dose from pQCT examinations is lower than $0.01 \mathrm{mSv}[9,14]$. In a recent study of pQCT applied to imaging of microstructure of the distal tibia in adolescents, Burrows et al. reported an effective dose for HR pQCT (XtremeCT, Scanco) lower than $0.003 \mathrm{mSv}$ [14]. Interestingly, these authors selected a measurement site so as to avoid primary irradiation of the growth plate. Dose optimisation is important even for low-dose methods as children and adolescents are more susceptible to the risk of radiation-induced biological effects than adults. However, the fact that both DXA and pQCT involve low radiation doses enables these techniques to be used to study the growth and development of the skeleton in normal children.

\section{Dose reduction techniques}

The system for patient radiation protection is based upon two principles: (a) justification and (b) optimisation. It is essential that all X-ray exposures used for estimation of bone status are clinically justified. Examinations that do not influence patient care must be avoided.

Patient preparation for bone densitometry is important for reducing the radiation dose. Careful checking for the presence of items on patient's clothing such as jewellery and coins that cause artefacts will optimise the quality of

Table 2 Typical effective and organ doses for various diagnostic X-ray examinations

\begin{tabular}{llll}
\hline Examination & Effective dose (mSv) & Organ dose (mGy) & Relevant organs \\
\hline Dental radiography (intraoral) & 0.005 & 0.005 & Brain \\
Chest radiography (posterior-anterior) & 0.02 & 0.01 & Lung \\
X-ray mammography & 0.4 & 3 & Breast \\
Adult abdominal CT & 8 & 10 & Stomach \\
\hline
\end{tabular}


Table 3 Radiation doses associated with QCT protocols

\begin{tabular}{|c|c|c|c|c|}
\hline Examination & Voltage $(\mathrm{kV})$ & X-ray tube load (mAs) & Effective dose (mSv) & References \\
\hline 2D QCT spine, scout image and 3 slices of $10-\mathrm{mm}$ thickness & 80 & 125 & $0.06-0.3$ & {$[30,41]$} \\
\hline 3D MDQCT spine, L1-L2, pitch 1 & 120 & 100 & 1.5 & [9] \\
\hline 3D MDQCT hip, pitch 1 & 120 & $150-200$ & $2.5-3.0$ & [9] \\
\hline 3D MDQCT radius, pitch 1 & 120 & 100 & $<0.01$ & [43] \\
\hline
\end{tabular}

Patient dose may differ significantly between institutions because of the variability in acquisition protocols and differences in the CT system characteristics

the imaging and avoid the imaging having to be repeated with additional radiation exposure. In paediatric examinations, proper interaction with the children and parents is essential. All actions should be taken to avoid movement of the child during imaging and defer imaging if artefacts (e.g. plaster of Paris on fractures) are present at the imaging site.

The length of the DXA or CT should be minimal and should take into account patient's body size. If possible, radiosensitive organs should not be exposed to the primary radiation beam. Modern MDCT employ multiple rows of detector arrays allowing rapid imaging. As a consequence, the length of imaging is sometimes increased to include multiple body sites. This increases the DLP and the patient effective dose.

After the introduction of multi-detector CT, significant attention was given to doses and associated risks from CT. In spiral $\mathrm{CT}$, reconstruction algorithms require a number of additional rotations to reconstruct the first and last slice of imaged volume. Z-overscanning refers to the extent of the tissue beyond the boundaries of the volume to be imaged that is exposed to X-rays. The increase in patient effective dose due to z-overscanning may reach $36 \%$ for a 16-slice CT system [47]. In hip MDQCT and HR MDCT imaging the influence of z-overscanning on the patient effective dose should always be taken into account. Careful selection of beam collimation, reconstructed image slice width and pitch is needed to limit the extent of z-overscanning [48]. A recent study showed that adaptive section collimation allows considerable dose reduction of unnecessary exposure due to z-overscanning [49].

Variation in the tube voltage causes a substantial change in patient $\mathrm{CT}$ dose and image quality [50]. The use of $80 \mathrm{kVp}$ for spinal single-slice QCT reduces the dose substantially in comparison with acquisition at $120 \mathrm{kVp}$. The possibility of tube voltage reduction in MDQCT should be investigated, especially for patients with small body size. Reduction of tube load may reduce patient dose considerably. Automatic exposure control (AEC) tools adjust the tube current in the $x-y$ plane and along $\mathrm{z}$ axis simultaneously, based on the size and attenuation of the anatomical area being examined to achieve an operator-defined level of image quality with the lowest possible dose. The use of AEC provides a substantial potential for dose reduction compared with the fixed $\mathrm{mA}$ technique. In AEC-activated examinations, the mAs product can be reduced by typically between $15 \%$ and $60 \%[51,52]$.

\section{Occupational radiation doses and shielding}

Several studies have evaluated scattered radiation from pencil-beam and fan-beam DXA systems and the occupational dose to operators $[34,53,54]$. The time-averaged dose rates at $1 \mathrm{~m}$ from the central axis of the imaging table range from about $0.01 \mu \mathrm{Sv} / \mathrm{h}$ to about $5 \mu \mathrm{Sv} / \mathrm{h}$, depending on the DXA model. Scatter radiation from fan-beam systems is higher than that from pencil-beam systems. The annual occupational dose at $1 \mathrm{~m}$ from a fan-beam system can reach $1.5 \mathrm{mSv}$ [34]. With regard to occupational doses, the ICRP recommends a limit on the effective dose of $20 \mathrm{mSv} /$ year, averaged over 5 years, with a further provision that the effective dose should not exceed $50 \mathrm{mSv}$ in any single year. Although the dose limit of $20 \mathrm{mSv} / y e a r$ is much higher than the expected annual occupational doses from DXA, the use of a protective lead shield may be necessary occasionally for fan-beam systems in a confined space to ensure that the operator dose is as low as possible [34].

Special dose limits apply for the conceptus after a pregnant employee declares pregnancy. The ICRP and European Commission recommend that the individual developing in utero should be protected by the application of a dose limit of $1 \mathrm{mGy}$ during the remainder of pregnancy, once it has been declared $[55,56]$. The exclusion of pregnant workers from DXA examinations on the basis of radiogenic risks from occupational DXA exposure cannot be justified on scientific grounds. However, the scatter radiation can exceed the exposure limits for pregnant workers, especially for fan-beam systems. For this reason, radiation protection measures should always be taken to ensure that the conceptus dose will be kept below $1 \mathrm{mGy}$ during the declared pregnancy. Moreover, the use of a personal radiation meter at waist level is recommended to monitor radiation exposure.

An important requirement in limiting the risk of radiation exposure in the workplace is the correct design of the room in which the imaging device has been installed. Measurements performed by Larkin et al. [34] showed that the scatter from fan-beam DXA systems can exceed the limits for public exposure i.e. $1 \mathrm{mSv} /$ year [19]. In these cases, additional structural shielding might be required, especially when the distance from the imaging table to the adjacent wall is less than $1 \mathrm{~m}$. However, other parameters should also be taken into account such as the workload, the material of the walls, the location of the operator and the location and use of rooms that adjoin the imaging room. 


\section{Radiogenic risks associated with techniques used in bone status evaluation}

Although diagnostic X-ray examinations provide great benefits, their use involves the potential risk of carcinogenesis. Estimation of cancer risks associated with the radiation exposure from diagnostic X-rays is possible by using radiation dose data and appropriate risk coefficients provided by scientific committees. Risk estimation is based on the linear no-threshold (LNT) model. LNT presupposes that there is a linear relationship between radiation dose and health risk at all dose levels. The risk estimate based on the LNT model is a useful tool not only for justification of medical exposures but also for comparison with other risks. The Biological Effects of Ionizing Radiation Committee VII (BEIR VII) recently estimated sex- and age-specific risk factors attributable to radiation [57]. Of note, however, is that patient and occupational effective doses from techniques used in clinical practice for evaluation of bone status and potential radiogenic risks are very small compared with the expected benefits [58].

Assessment of radiogenic risk from diagnostic X-ray examinations has several limitations. There is debate regarding whether low-level radiation provides a signifi- cantly increased risk of developing cancer. The biological effects of diagnostic radiation on humans have not been studied directly. Current information is based on studies of populations such as atomic bomb survivors, radiation workers and patients treated with radiation. Most individuals in these cohorts received effective doses that were much larger than the doses from diagnostic X-ray examinations. However, recent studies show a significant increase in cancer risk in a group of atomic bomb survivors who received doses of radiation comparable to those currently involved in diagnostic radiology [48, 59]. The potential risk to an individual associated with techniques used for the assessment of bone status is very small because radiation doses are low. Despite uncertainty about the true risks of exposure to radiation levels used in these techniques, radiation protection principles must be applied in everyday clinical practice.

Open Access This article is distributed under the terms of the Creative Commons Attribution Noncommercial License which permits any noncommercial use, distribution, and reproduction in any medium, provided the original author(s) and source are credited.

\section{References}

1. Compston JE, Papapoulos SE, Blanchard F (1998) Report on osteoporosis in the European Community: current status and recommendations for the future. Osteoporos Int 8:531-534

2. Gehlbach SH, Bigelow C, Heimisdottir $M$ et al (2000) Recognition of vertebral fracture in a clinical setting. Osteoporos Int 11:577-582

3. Genant HK, Wu CY, van Kuijk C, Nevitt MC (1993) Vertebral fracture assessment using a semiquantitative technique. J Bone Miner Res 8:1137-1148

4. Jiang G, Eastell R, Barrington NA, Ferrar L (2004) Comparison of methods for the visual identification of prevalent vertebral fracture in osteoporosis. Osteoporos Int 15:887-896

5. Guglielmi G, Diacinti D, van Kuijk C et al (2008) Vertebral morphometry: current methods and recent advances. Eur Radiol 18:1484-1496

6. Guglielmi G, Palmieri F, Placentino M, D' Errico F, Stoppino L (2009) Assessment of osteoporotic vertebral fractures using specialized workflow software for 6-point morphometry. Eur J Radiol 70:142-148
7. Fuerst T, Wu C, Genant HK et al (2009) Evaluation of vertebral fracture assessment by dual X-ray absorptiometry in a multicenter setting. Osteoporos Int 20:1199-1205

8. Lenchik L, Rochmis P, Sartoris D (1998) Optimized interpretation and reporting of dual X-ray absorptiometry (DXA) scans. AJR Am J Roentgenol 171:1509-1520

9. Engelke K, Adams JE, Armbrecht G et al (2008) Clinical use of quantitative computed tomography and peripheral quantitative computed tomography in the management of osteoporosis in adults: the 2007 ISCD official positions. J Clin Densitom 11:123162

10. Borggrefe J, Graeff C, Nickelsen TN, Marin F, Glüer CC (2009) Quantitative computed tomography assessment of the effects of 24 months teriparatide treatment on 3D femoral neck bone distribution, geometry and bone strength: results from the EUROFORS study. J Bone Miner Res. doi:10.1359/ jbmr.090820

11. Issever A, Link T, Kentenich $M$ et al (2009) Assessment of trabecular bone structure using MDCT: comparison of 64-and 320-slice CT using HR-pQCT as the reference standard. Eur Radiol 20:458-468
12. Krebs A, Graeff C, Frieling I et al (2009) High resolution computed tomography of the vertebrae yields accurate information on trabecular distances if processed by 3D fuzzy segmentation approaches. Bone $44: 145-152$

13. Bacchetta J, Boutroy S, Vilayphiou N et al (2009) Early impairment of trabecular microarchitecture assessed with HRpQCT in patients with stage II-IV chronic kidney disease. J Bone Miner Res. doi:10.1359/jbmr.090831

14. Burrows M, Liu D, McKay H (2009) High resolution peripheral QCT imaging of bone micro-structure in adolescents. Osteoporos Int. doi:10.1007/s00198-009-0913-2

15. Intenzo CM, Parker L, Rao VM, Levin DC (2005) Changes in procedure volume and service provider distribution among radiologists and nonradiologists in dual-energy X-ray absorptiometry between 1996 and 2002 . J Am Coll Radiol 2:662-664

16. Curtis JR, Carbone L, Cheng $\mathrm{H}$ et al (2008) Longitudinal trends in use of bone mass measurement among older Americans, 1999-2005. J Bone Miner Res 23:1061-1067 
17. Nelson HD, Helfand M, Woolf SH, Allan JD (2002) Screening for postmenopausal osteoporosis: a review of the evidence for the US Preventive Services Task Force. Ann Intern Med 137:529-541

18. National Osteoporosis Foundation (1998) Physician's guide to prevention and treatment of osteoporosis. Excerpta Medica, Belle, Mead

19. Recommendations of the International Commission on Radiological Protection [ICRP publication 103] (2007) Ann ICRP 37:1-332

20. Kalender WA (2005) Computed tomography. Publicis, Erlangen

21. Managing patient dose in multi-detector computed tomography (MDCT) [ICRP Publication 102] (2007) Ann ICRP 37:1-79

22. Vokes T, Bachman D, Baim S et al (2006) Vertebral fracture assessment: the 2005 ISCD official positions. J Clin Densitom 9:37-46

23. Blake GM, Rea JA, Fogelman I (1997) Vertebral morphometry studies using dual-energy X-ray absorptiometry. Semin Nucl Med 27:276-290

24. Ferrar L, Jiang G, Adams J, Eastell R (2005) Identification of vertebral fractures: an update. Osteoporos Int 16:717-728

25. Grados F, Fechtenbaum J, Flipon E, Kolta S, Roux C, Fardellone P (2009) Radiographic methods for evaluating osteoporotic vertebral fractures. Joint Bone Spine 76:241-247

26. Bauer JS, Muller D, Ambekar A et al (2006) Detection of osteoporotic vertebral fractures using multidetector CT. Osteoporos Int 17:608-615

27. Bezakova E, Collins PJ, Beddoe AH (1997) Absorbed dose measurements in dual energy X-ray absorptiometry (DXA). Br J Radiol 70:172-179

28. Lewis MK, Blake GM, Fogelman I (1994) Patient dose in dual X-ray absorptiometry. Osteoporos Int 4:11-15

29. Cawte SA, Pearson D, Green DJ, Maslanka WB, Miller CG, Rogers AT (1999) Cross-calibration, precision and patient dose measurements in preparation for clinical trials using dual energy X-ray absorptiometry of the lumbar spine. Br J Radiol 72:354-362

30. Huda W, Morin RL (1996) Patient doses in bone mineral densitometry. $\mathrm{Br}$ J Radiol 69:422-425

31. Njeh CF, Samat SB, Nightingale A, McNeil EA, Boivin CM (1997) Radiation dose and in vitro precision in bone mineral density measurement using dual X-ray absorptiometry. Br J Radiol 70:719-727

32. Steel SA, Baker AJ, Saunderson JR (1998) An assessment of the radiation dose to patients and staff from a Lunar Expert-XL fan beam densitometer. Physiol Meas 19:17-26
33. Blake G, Naeem M, Boutros M (2006) Comparison of effective dose to children and adults from dual X-ray absorptiometry examinations. Bone 38:935-942

34. Larkin A, Sheahan N, O'Connor U et al (2008) QA/Acceptance testing of DEXA X-ray systems used in bone mineral densitometry. Radiat Prot Dosim 129:279-283

35. Brenner D, Hall E (2007) Computed tomography - an increasing source of radiation exposure. N Engl J Med 357:2277-2284

36. Mettler F, Huda W, Yoshizumi T, Mahesh M (2008) Effective doses in radiology and diagnostic nuclear medicine: a catalog. Radiology 248:254-263

37. Faulkner RA, Bailey DA, Drinkwater DT et al (1993) Regional and total body bone mineral content, bone mineral density and total body tissue composition in children 8-16 years of age. Calcif Tissue Int 53:7-12

38. Thorpe JA, Steel SA (2006) The DXA calscan heel densitometer: evaluation and diagnostic thresholds. Br J Radiol 79:336-341

39. Damilakis J, Perisinakis K, Vrahoriti H, Kontakis G, Varveris H, Gourtsoyiannis N (2002) Embryo/fetus radiation dose and risk from dual X-ray absorptiometry examinations. Osteoporos Int 13:716722

40. Recommendations of the International Commission on Radiological Protection [ICRP publication 84] (2000) Ann ICRP 30:1-43

41. Kalender WA, Buchenau S, Deak P et al (2008) Technical approaches to the optimisation of CT. Phys Med 24:71-79

42. Kalender WA (1992) Effective dose values in bone mineral measurements by photon absorptiometry and computed tomography. Osteoporos Int 2:82-87

43. Khoo BC, Brown K, Cann $\mathrm{C}$ et al (2009) Comparison of QCT-derived and DXA-derived areal bone mineral density and T scores. Osteoporos Int 20:1539-1545

44. Engelke C, Libanati C, Liu Y et al (2009) Quantitative computed tomography (QCT) of the forearm using general purpose spiral whole-body CT scanners: accuracy, precision and comparison with dual-energy X-ray absorptiometry (DXA). Bone 45:110 118

45. Ito $\mathrm{M}$, Ikeda $\mathrm{K}$, Nishiguchi $\mathrm{M}$ et al (2005) Multidetector row CT imaging of vertebral microstructure for evaluation of fracture risk. J Bone Miner Res 20:1828-1836

46. Graeff C, Timm W, Nickelsen TN, Farrerons J et al (2007) Monitoring teriparatide-associated changes in vertebral microstructure by highresolution CT in vivo: results from the EUROFORS study. J Bone Miner Res 22:1426-1433
47. Tzedakis A, Damilakis J, Perisinakis K, Stratakis J, Gourtsoyiannis N (2005) The effect of $\mathrm{z}$ overscanning on patient effective dose from multidetector helical computed tomography examinations. Med Phys 32:1621-1629

48. Pierce DA, Preston DL (2000) Radiation-related cancer risks at low doses among atomic bomb survivors. Radiat Res 154:178-186

49. Deak PD, Langner O, Lell M, Kalender WA (2009) Effects of adaptive section collimation on patient radiation dose in multisection spiral CT. Radiology 252:140-147

50. Kalender WA, Deak P, Kellermeier M, van Straten M, Vollmar SV (2009) Application- and patient size-dependent optimization of X-ray spectra for CT. Med Phys 36:993-1007

51. Papadakis AE, Perisinakis K, Damilakis J (2008) Automatic exposure control in pediatric and adult multidetector CT examinations: a phantom study on dose reduction and image quality. Med Phys 35:4567-4576

52. Lechel U, Becker C, Langenfeld-Jager G, Brix G (2009) Dose reduction by automatic exposure control in multidetector computed tomography: comparison between measurement and calculation. Eur Radiol 19:1027-1034

53. Patel R, Blake GM, Batchelor S, Fogelamn I (1996) Occupational dose to the radiographer in dual X-ray absorptiometry: a comparison of pencilbeam and fan-beam systems. Br J Radiol 69:539-543

54. Njeh CF, Apple K, Temperton DH, Boivin CM (1996) Radiological assessment of a new bone densitometer - the Lunar Expert. Br J Radiol 69:335-340

55. International Commission on Radiological Protection [ICRP publication 84] (2000) Pregnancy and medical radiation, Pergamon

56. European Commission [EU publication RP 100] (1998) Guidance for protection of unborn children and infants irradiated due to parental medical exposures. Official Publications of the European Communities

57. Committee to Assess Health Risks from Exposure to Low levels of Ionizing Radiation, Nuclear and Radiation Studies Board, Division on Earth and Life Studies, National Research Council of the National Academies (2006) Health risks from exposure to low levels of ionizing radiation: BEIR VII Phase 2. The National Academy Press, Washington

58. Njeh CF, Fuerst T, Hans D, Blake G, Genant HK (1999) Radiation exposure in bone mineral density assessment. Appl Radiat Isot 50:215-236

59. Preston DL, Ron E, Tokuoka S et al (2007) Solid cancer incidence in atomic bomb survivors: 1958-1998. Radiat Res 168:1-64 